\title{
Combined Effects of Blood Pressure and Aldosterone on Cardiac Left Ventricular Mass Index-Ethnic Differences between Kazakh, Uygur and Han Subjects
}

\author{
Masakatsu Ohta1, Yuji Kasamaki², Yukio Ozawa1, Atsushi Hirayama1, \\ Tomohiro Nakayama ${ }^{3}$, Hiroshi Kawamura ${ }^{4}$, Dilxat Himit ${ }^{5}$, \\ Masayoshi Soma ${ }^{2}$, Yoichi Izumí \\ ${ }^{1}$ Division of Cardiology, Department of Medicine, Nihon University School of Medicine, Tokyo, Japan \\ ${ }^{2}$ Division of General Medicine, Department of Medicine, Nihon University School of Medicine, Tokyo, Japan \\ ${ }^{3}$ Division of Laboratory Medicine, Department of Pathology and Microbiology, Nihon University School of \\ Medicine, Tokyo, Japan \\ ${ }^{4}$ Department of Internal Medicine, Nippon Dental University, Tokyo, Japan \\ ${ }^{5}$ Department of Science and Research, Xinjiang Medical University, Urumqi, China \\ ${ }^{6}$ Department of Cardiology, Kanazawa Medical University, Himi Municipal Hospital, Himi, Japan \\ Email: kasamaki.yuuji@nihon-u.ac.jp
}

Received 19 December 2013; revised 25 January 2014; accepted 2 March 2014

Copyright (C) 2014 by authors and Scientific Research Publishing Inc.

This work is licensed under the Creative Commons Attribution International License (CC BY).

http://creativecommons.org/licenses/by/4.0/

c) (i) Open Access

\section{Abstract}

Previous Background: Hemodynamic factors, like blood pressure, have been established to be major determinants of cardiac left ventricular structure. However, several factors other than blood pressure to influence cardiac mass have been implicated. When we did medical survey, cardiac left ventricular mass index (LVMI) of one ethnic group that had higher blood pressure was found to be smaller than that of the other ethnic groups with a lower blood pressure. Such contradicted data from the present study were analyzed combining blood pressure, LVMI and chemical parameters obtained from blood and urine. Methods: In a medical survey conducted in Xinjiang, China, 279 people (65 - 70 years old) from three ethnic groups (Kazakh, Uygur and Han) from two separated regions provided blood and urine samples and underwent echocardiography and 24-h ambulatory blood pressure monitoring (ABPM). Results: Systolic and diastolic blood pressure obtained from ABPM and urinary sodium excretion values were significantly higher in Kazakh than that in Uygur and Han. However, LVMI in Kazakh was lower than that in other 2 groups. Plasma aldosterone concentration (PAC) and plasma renin activity (PRA) were significantly lowest in Kazakh. The values of LVMI in all ethnic groups were positively related to both blood pressure and PAC. An in- 
verse correlation was identified between PAC and urinary sodium excretion value. Conclusion: Although higher blood pressure in Kazakh subjects, their LVMI was lower than those of Uygur and Han, whose blood pressure was lower than that in Kazakh. These results suggest that blood pressure is not always a determinant for LVMI value. There is a possibility that relatively lower PAC resulted from higher sodium intake suppressed the rise in LVMI caused by higher blood pressure in Kazakh.

\section{Keywords}

\section{Left Ventricular Mass; Aldosterone; Blood Pressure; Salt Intake}

\section{Introduction}

Morphological changes in the heart during development of hypertension are an important issue, as blood pressure is a major factor in cardiac hypertrophy [1] [2]. However, clinical and experimental studies have revealed that several factors other than blood pressure load participate in the pathogenesis of cardiac hypertrophy [3] [4], as well as discrepancies between blood pressure control and degree of cardiac hypertrophy [5] [6]. For example, several antihypertensive agents have been found to promote the regression of cardiac hypertrophy without hypotensive action [7]-[9]. Such suppressive effects by antihypertensive agents on cardiac hypertrophy without inhibition of hemodynamic load result from direct action against angiotensin II or catecholamine receptors. On the other hand, it has been well established that the aldosterone receptor antagonists are also useful for the regression of cardiac hypertrophy [10]. Low doses of this agent induce protective effects on the development of cardiac hypertrophy without reducing blood pressure [11], thus suggesting that aldosterone is one of the factors that stimulate enlargement of cardiac mass.

The data obtained from elderly subjects in a previous survey revealed conflicting results, in which cardiac left ventricular index (LVMI) in an ethnic group with higher blood pressure was found to be smaller than that in other ethnic groups with lower blood pressure. Such contradictory data were analyzed by combining blood pressure, LVMI and chemical parameters obtained from blood and urine to determine whether parameters other than blood pressure participate in the control of LVMI.

\section{Subjects and Methods}

A total of 310 healthy adults from 3 ethnic groups were recruited by random selection of resident cards from local government offices in Hotan (Hotan Prefecture, southern part of Xinjiang in China) and Balikun (Balikun Prefecture, northeastern part of Xinjiang in China). The Ethics Committee of Xinjiang Medical University approved all study protocols. All subjects provided informed consent before participating in this study. Although many subjects were chosen at random, some of the subjects were excluded for reasons as follows; their age were not fit an account book, records of ABPM were not produced precisely, and collection of urine was incomplete. Thus, a large difference has been produced to a man and woman. A total of 279 subjects were enrolled in this study, after excluding subjects with incomplete data. No subjects had previously received antihypertensive agents or medication. Subjects from the 3 ethnic groups (elderly Kazakh subjects in Balikun: age 65 to70 years, $\mathrm{n}=100$, 39 males, 61 females; elderly Uygur subjects in Hotan: age 65 to 70 years, $n=94,62$ males, 32 females; elderly Han subjects in Hotan and Balikun: age 65 to 70 years, $n=85$, 44 males, 41 females) were hospitalized for 4 days at local medical centers in Hotan or Balikun. The Han subjects who participated in the study lived in both regions. Therefore, since these subjects were of the same ethnic background but lived in different regions, this allowed us to examine whether environmental differences were responsible for any of noted changes that might be found during the study.

Subjects arrived at the medical centers in the evening of the first day of hospitalization. All subjects underwent a physical examination, including measurement of body weight and height.

On the morning of the second day of hospitalization, 24-h urine collection was initiated in order to determine urine volume and sodium excretion levels. Venous blood was drawn on day 3, after the end of the urine collection cycle and before breakfast, in order to determine PRA and PAC. Subsequently, echocardiographic examina- 
tion was carried out. ABPM using a TM 2421 monitor (A\&D Corp., Tokyo, Japan) was performed. From echocardiographic examination, diastolic thickness of the interventricular septum (IVSTd mm), left ventricular posterior wall (PWTd mm) and left ventricular diastolic dimensions (LVDd mm) were measured from a parasternal left ventricular long axis view and a short axis view. Left ventricular mass (LVM) was calculated using the formula: $\mathrm{LVM}=1.04$ [(IVSTd + PWTd + LVDd $\left.)^{3}-\mathrm{LVDd}^{3}\right]-13.6$, as reported previously [12]. Left ventricular mass index (LVMI) was corrected for body surface area (BSA g/ $\mathrm{m}^{2}$ ). Mean systolic blood pressure (SBP) and mean diastolic blood pressure (DBP) obtained during 24-h ABPM were used as blood-pressure values in the study. The subjects allowed to act freely in the hospital except for the meal, blood sampling and installation of ABPM. Traditional Islamic meals were provided during hospitalization. Urinary sodium levels were measured using an RA-2000 automatic analyzer (Technicon Instruments, Tarrytown, NY, USA). Radioimmunoassay kits were used to measure PRA (Beijing North Biotechnology Institute, Beijing, China), PAC (Diagnostic Products Corp., Los Angeles, CA, USA). Body mass index (BMI) was calculated as follows: BMI = BW/(height, in meters $)^{2}$. BSA was calculated as follows: BSA $=\left(\right.$ weight $^{0.425} \times$ height $\left.^{0.725}\right) \times 0.007184$.

\section{Statistical Analysis}

All values are shown as means \pm standard error (SE). All statistical calculations were performed using SPSS for Windows (version 11.5; SPSS, Chicago, IL, USA). Intra-group comparisons were performed using one-way ANOVA with Turkey's post-hoc testing. Multiple regression analysis was performed using LVMI as a dependent variable and age, sex, BMI, SBP, PRA, PAC, urinary sodium excretion values and ethnicity as independent variables. Values of $\mathrm{P}<0.05$ were considered to be statistically significant.

\section{Results}

SBP and DBP were significantly higher in Kazakh subjects $(136.7 \pm 2.08 \mathrm{mmHg}$ and $77.9 \pm 1.12 \mathrm{mmHg})$ than in Uygur subjects $(125.2 \pm 1.73 \mathrm{mmHg}$ and $71.1 \pm 1.58 \mathrm{mmHg})$ and Han subjects $(127.2 \pm 2.11 \mathrm{mmHg}$ and 73.8 $\pm 1.12 \mathrm{mmHg}$; Figures 1(a), (b)). LVMI was significantly lower in Kazakh subjects $\left(84.7 \pm 2.29 \mathrm{~g} / \mathrm{m}^{2}\right)$ than in Uygur subjects $\left(98.3 \pm 2.92 \mathrm{~g} / \mathrm{m}^{2}\right)$ and Han subjects $\left(93.7 \pm 3.14 \mathrm{~g} / \mathrm{m}^{2}\right.$; Figure $\left.1(\mathrm{c})\right)$. Urinary sodium excretion values were significantly lower in Uygur subjects $(128.2 \pm 5.59 \mathrm{mEq} /$ day) than that in Kazakh subjects (178.8 \pm $7.75 \mathrm{mEq} /$ day) or Han subjects (168.1 $\pm 8.10 \mathrm{mEq} /$ day; Figure 1(d)). PRA was significantly lower in Kazakh subjects $(0.27 \pm 0.07 \mathrm{ng} / \mathrm{ml} / \mathrm{h})$ than in Uygur subjects $(0.58 \pm 0.03 \mathrm{ng} / \mathrm{ml} / \mathrm{h}$; Figure $1(\mathrm{e}))$ or Han subjects $(0.42 \pm$ $0.05 \mathrm{ng} / \mathrm{ml} / \mathrm{h})$. PAC was lowest in Kazakh subjects $(29.8 \pm 2.18 \mathrm{pg} / \mathrm{ml})$ in 3 groups. PAC in Han subjects $(75.3 \pm$ $6.84 \mathrm{pg} / \mathrm{ml}$ ) was lower than that in Uygur subjects (110.6 $\pm 5.58 \mathrm{pg} / \mathrm{ml}$ ) (Figure 1(f)).

When data from the 3 groups were analyzed together using multiple regression analysis, LVMI was correlated with SBP $(r=0.22, \mathrm{P}<0.0001$; Figure 2(a)), DBP $(r=0.18, \mathrm{P}<0.05$; Figure 2(b)) and PAC $(r=0.34, \mathrm{P}<$ 0.0001; Figure 2(c)). PAC was inversely correlated with urinary sodium excretion values $(\mathrm{r}=0.33, \mathrm{P}<0.05$; Figure 2(d)). PRA was not correlated with blood pressure, LVMI or PAC. Clinical characteristics of study subjects from the 3 ethnicities are shown in Table 1 . There was no difference in age between the 3 groups. BMI was highest in Kazakh. In multivariable linear regression analysis with LVMI as dependent variable, SBP $(\beta=$ $0.271, P=0.001)$ and PAC ( $\beta=0.183, P=0.01)$ were independently associated with LVMI (Table 2$)$.

For the Han subjects that lived either in the Hotan or in the Balikun regions, there were no differences noted for the BMI, SBP, DBP, age, GFR and LVMI. Urialy sodium excretion was higher in the Han subjects lived in Blikun than that in Hotan. PAC was lower in the Han subjects of Balikun than that of Hotan (data not shown).

\section{Discussion}

Numerous factors play a role in increasing LVMI. However, blood pressure has been identified as the major determinant in developing LVMI [13]. On the other hand, it has been reported that several chemical and neurological substances also influence cardiac LVMI [14]. For example, subclinical doses of antihypertensive agents, angiotensin-converting enzyme inhibitor [15], $\alpha 1$-blockade [8] and $\beta$-blockade are reported to be effective in preventing development of cardiac hypertrophy [16]. Low doses of spironolactone also protect against increases in LVMI without decreasing blood pressure [17], thus suggesting that aldosterone is an important substance for inducing enlargement of LVMI.

In the present survey, blood pressure was significantly higher in Kazakh than Uygur and Han subjects. In 


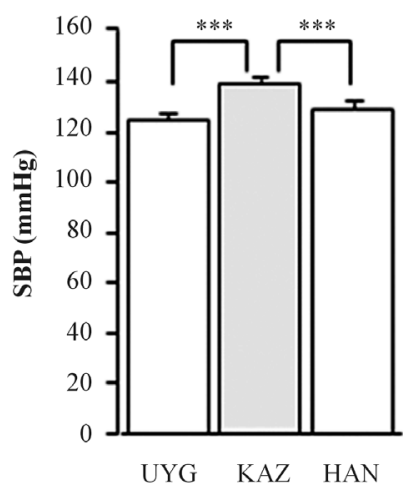

(a)

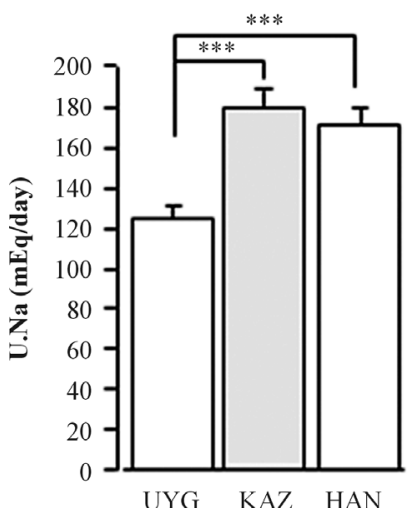

(d)

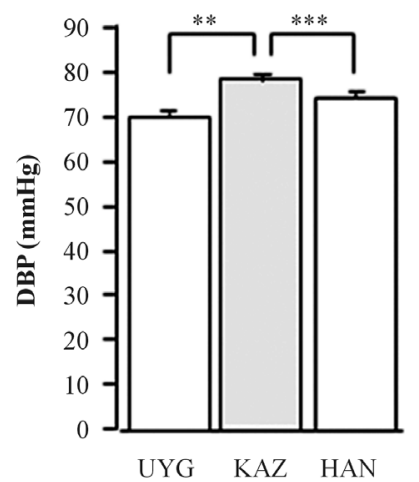

(b)

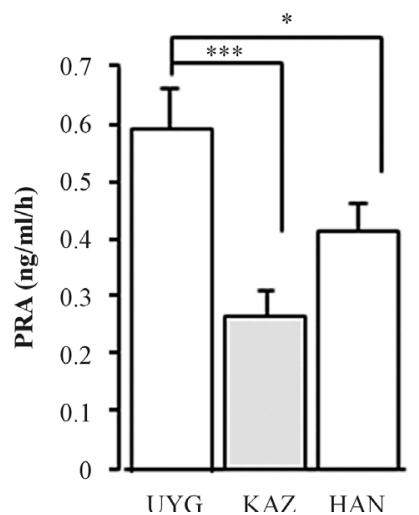

(e)

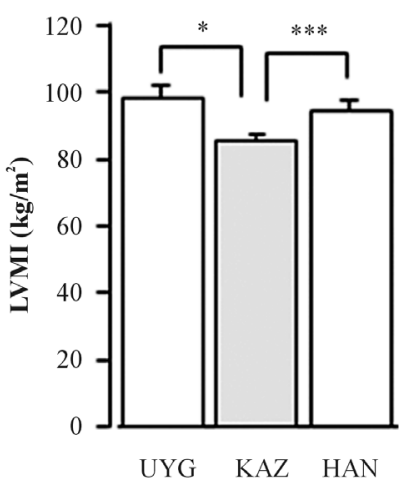

(c)

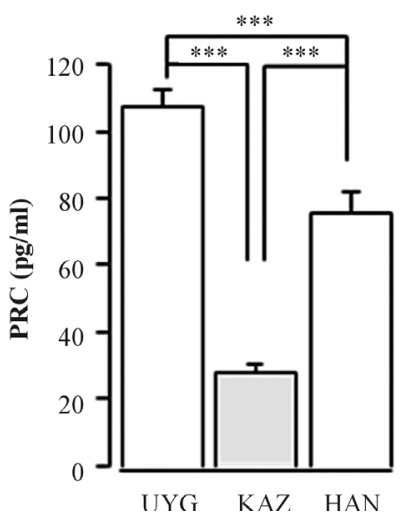

(f)

Figure 1. Systolic blood pressure (SBP, a) and diastolic blood pressure (DBP, b), as measured by ABPM, left ventricular mass (LVMI; c), urinary sodium excretion value (U.Na, d), plasma aldosterone concentration (PAC, e) and plasma renin activity (PRA, f) in the 3 ethnic groups. KAZ, Kazakh subjects; UYG, Uygur subjects; Han, Hun subjects.

Table 1. Comparison of demographic and biochemical characteristics between the study groups.

\begin{tabular}{ccccc}
\hline & KAZ & UYG & HAN & \\
\hline Variables & $(\mathrm{n}=100, \mathrm{~m} / \mathrm{f}=39 / 61)$ & $(\mathrm{n}=94, \mathrm{~m} / \mathrm{f}=62 / 32)$ & $(\mathrm{n}=85, \mathrm{~m} / \mathrm{f}=44 / 41)$ & $P$ \\
Age (years) & $67.1 \pm 0.17$ & $67.1 \pm 0.11$ & $67.2 \pm 0.20$ & NS \\
BMI $\left(\mathrm{kg} / \mathrm{m}^{2}\right)$ & $24.2 \pm 0.38^{*}$ & $21.1 \pm 0.23^{*}$ & $22.6 \pm 0.30^{*}$ & ${ }^{*} P<0.001$ vs. other groups \\
LVMI (g/m $)$ & $84.7 \pm 2.29$ & $98.3 \pm 2.29$ & $93.7 \pm 3.14$ & ${ }^{*} P<0.05$ vs.KAZ \\
SBP (mmHg) & $136.7 \pm 2.08$ & $125.2 \pm 1.73^{*}$ & $127.2 \pm 1.65^{*}$ & ${ }^{*} P<0.001$ vs.KAZ \\
DBP (mmHg) & $77.9 \pm 1.12^{*}$ & $71.1 \pm 1.58^{*}$ & $73.8 \pm 1.12^{*}$ & ${ }^{*} P<0.01$ vs. other groups \\
PRA (ng/ml/h) & $0.27 \pm 0.07$ & $0.58 \pm 0.03$ & $0.42 \pm 0.05$ & ${ }^{*} P<0.001$ vs. other groups \\
PAC (pg/ml) & $29.1 \pm 2.18$ & $110.6 \pm 5.58$ & $75.3 \pm 6.84$ & ${ }^{*} P<0.01$ vs. other groups \\
U. Na (mEq/day) & $178.8 \pm 7.75^{*}$ & $128.2 \pm 5.59$ & $168.1 \pm 8.10^{*}$ & ${ }^{*} P<0.0001$ vs.UYG \\
\hline
\end{tabular}

Values are expressed as mean \pm SE. KAZ, Kazakh people; UYG, Uygur people; HAN indicates Han people; m/f, male/female; BMI, body mass index; LVMI, left ventricular mass index; SBP, systolic blood pressure; DBP, diastolic blood pressure; PRA, plasma renin activity; PAC, plasma aldosterone concentration; U.Na, urinary sodium excretion.

contrast, LVMI was significantly lowest in Kazakh subjects. Higher LVMI in Kazakh subjects was expected, as they tend to have higher blood pressure. Therefore, factors other than blood pressure that may influence LVMI were examined in the present study. Large differences in PAC and PRA were seen between Kazakh subjects and 
Table 2. Multiple regression analysis with LVMI as the dependent variable.

\begin{tabular}{ccccc}
\hline & \multicolumn{3}{c}{ LVMI $\left(\mathrm{g} / \mathrm{m}^{2}\right)$} & \\
\hline & SE & $\beta$ & $t$ & 0.1020 \\
\hline sex (male/female) & 3.858 & -0.115 & -1.846 & 0.2150 \\
age (years) & 0.951 & -0.080 & -1.279 & 0.4950 \\
BMI (kg/m²) & 0.552 & -0.046 & -0.110 & 0.0001 \\
SBP (mmHg) & 0.095 & 0.271 & 3.906 & 0.5310 \\
PRA (ng/ml/h) & 3.052 & -0.040 & -0.675 & 0.0100 \\
PAC (pg/ml) & 0.035 & 0.183 & 2.900 & 0.2480 \\
U.Na (mEq/day) & 0.029 & -0.081 & -1.002 & 0.9070 \\
Ethnicity & 2.206 & 0.008 & 0.319 & \\
\hline
\end{tabular}

LVMI, left ventricular mass index; BMI, body mass index; SBP, systolic blood pressure; PRA, plasma renin activity; PAC, plasma aldosterone concentration; U.Na, urinary sodium excretion.

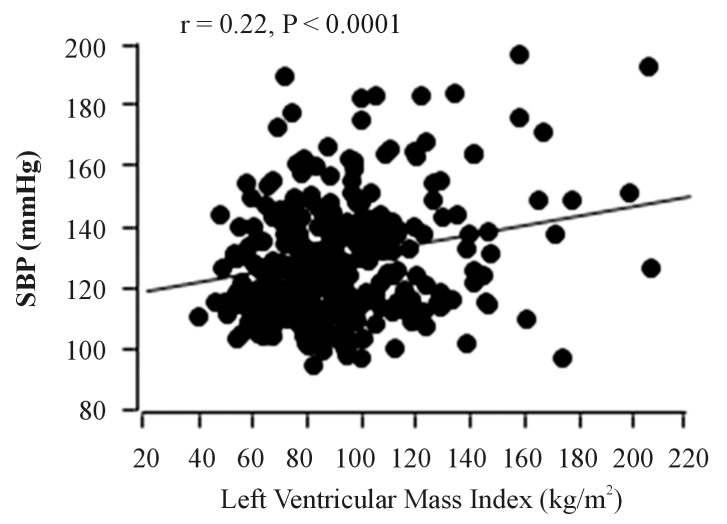

(a)

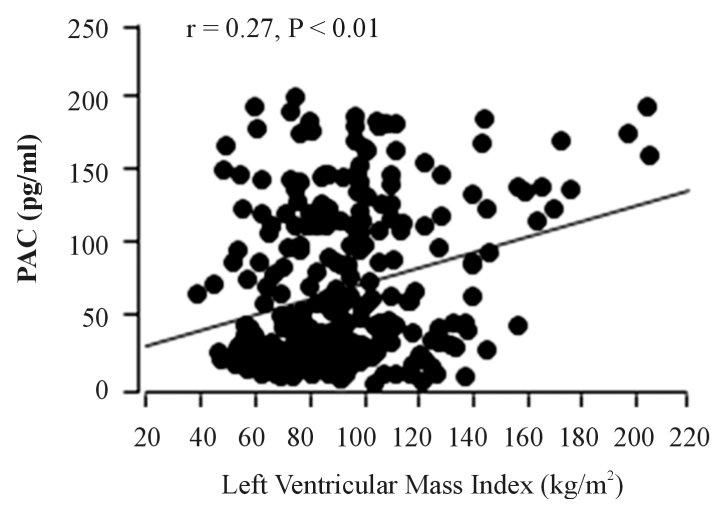

(c)

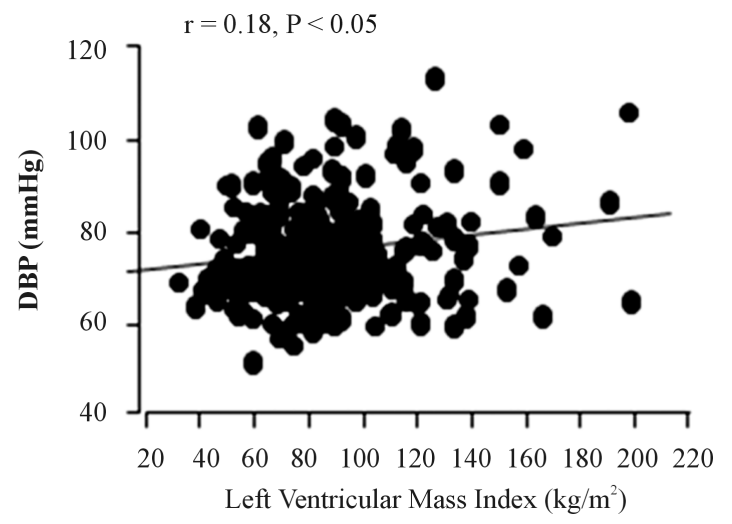

(b)

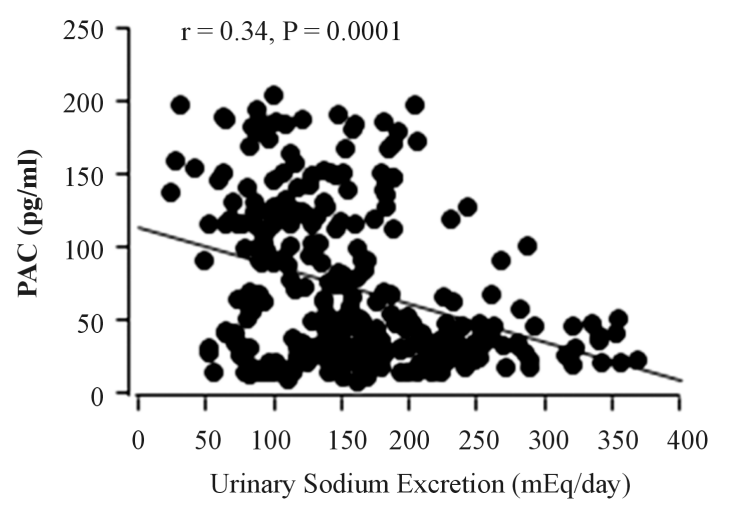

(d)

Figure 2. Relationship between left ventricular mass (LVMI) and systolic blood pressure (SBP, a), diastolic blood pressure (DBP, b), as measured by ABPM, and plasma aldosterone concentration (PAC; $\mathrm{c}$ ), and between PAC and urinary sodium excretion value (U. Na; d). The 3 ethnic groups were combined and relationships were evaluated by multivariate analysis.

Uygur subjects, as shown in Figures $\mathbf{1 ( e )}$ and (f). As the renin-angiotensin-aldosterone system is known to influence LVMI, it appears that lower PAC and PRA have a negative effect on development of LVMI.

The present results confirmed the relationship between blood pressure and LVMI, as shown in Figure 2(a) 
and (b), in which multiple liner regression analysis between blood pressure and LVMI in the 3 ethnic groups clearly showed a positive relationship. Therefore, blood pressure is still a major factor affecting LVMI. In addition, PAC was significantly correlated with LVMI, as shown in Figure 2(c), thus confirming that aldosterone is also an important factor for determining LVMI. In contrast, PRA was unrelated to LVMI, and there were no significant correlations between PAC and PRA. Although a close correlation between renin and aldosterone is known, the absence of a relationship between PAC and PRA in the present study cannot be explained.

The inverse correlation of PAC and urinary sodium excretion values in the present study was as expected. Higher salt intake reflected by higher urinary sodium excretion in Kazakh subjects apparently induced their low PAC. Urinary sodium excretion values were associated with blood pressure, but not with LVMI (data not shown). When these findings are generalized, dietary sodium plays a role in modulating LVMI indirectly through blood pressure and PAC, although it did not directly influence LVMI. High salt intake induces higher blood pressure, which causes higher LVMI [18]. On the other hand, high salt intake reduces PAC. Therefore, even if blood pressure was higher in Kazakh subjects, LVMI was lower than in Uygur subjects, who showed higher PAC than Kazakh subjects. In other words, decreased PAC increased LVMI via higher blood pressure caused by higher salt intake, which in turn lowered PAC. Confusing relationships between left ventricular structure and urinary sodium excretion and aldosterone have been reported [19]. Based on the present study, the changes in aldosterone and blood pressure may have had the opposite effect on LVMI. High salt intake causes increased blood pressure and LVMI, and decreasing aldosterone, possibly protecting against enlargement of LVMI. However, the potential interaction between PAC and blood pressure should also be considered in determining the association of aldosterone, blood pressure and LVMI.

A limitation of this study was that there were no significant associations between PRA, urinary sodium excretion values and LVMI. The final activating factor in the renin-angiotensin system is angiotensin II (A-II). However, the status of A-II was not determined, either in circulation or locally. A-II is a powerful stimulating factor in aldosterone production. It is possible that if this polypeptide was examined in this study, a significant association between A-II and LVMI may be seen. This polypeptide should therefore be examined to determine whether it is significantly associated with LVMI.

\section{Conclusion}

In conclusion, the present results indicate that both blood pressure and aldosterone are direct determinants of LVMI. Salt intake regulated these determinants, inducing increases in blood pressure and decreases in aldosterone. The changes in these parameters in this study have a counter effect on LVMI, depending on changes in salt intake, thus suggesting a complex relationship between salt intake and LVMI. Thus, the higher blood pressure and lower LVMI in Kazakh subjects can be explained by variations in blood pressure, PAC and urinary sodium excretion.

\section{References}

[1] Swynghedauw, B. and Delcayre, C. (1982) Biology of Cardiac Overload. Pathobiology Annual, 12, 137-183.

[2] Tarazi, R.C. and Levy, M.N. (1982) Cardiac Response in Increased Afterload: State-of-the-Art Review. Hypertension, 4, 8-18.

[3] Frdhlich, E.D. and Tarazi, R.C. (1976) Is Arterial Pressure the Sole Factor Responsible for Hypertensive Heart Hypertrophy? American Journal of Cardiology, 44, 956-963.

[4] Morgan, H.E. and Baker, K.M. (1991) Cardiac Hypertrophy: Mechanical, Neural and Endocrine Dependence. Circulation, 83, 13-25. http://dx.doi.org/10.1161/01.CIR.83.1.13

[5] Scuteri, A., Cacciafesta, M., Di Bernardo, M.G., De Propris, A.M., Recchi, D., Celli, V., Casagrande-Raffi, I., Sinopoli, C. and Marigliano, V. (1996) Is Blood Pressure the Major Determinant of Left Ventricular Mass in Subjects over 50 Years of Age? Archives of Gerontology and Geriatrics, 22, 181-194. http://dx.doi.org/10.1016/0167-4943(95)00692-3

[6] Drayer, J.I.M., Gardin, J.M., Weber, M.A. and Aronow, W.S. (1983) Cardiac Muscle during Vasodilation Therapy of Hypertension. Clinical Pharmacology \& Therapeutics, 33, 727-732. http://dx.doi.org/10.1038/clpt.1983.99

[7] Dunn, F.G., Oigman, W., Ventora, H.O., Messerli, F.H., Kobrin, I. and Frohlich, E.D. (1984) Enalapril Improves Systemic and Hemodynamics and Allows Regression of Left Ventricular Mass in Essential Hypertension. American Journal of Cardiology, 53, 105-108. http://dx.doi.org/10.1016/0002-9149(84)90692-1

[8] Schelling, P., Fischer, H. and Ganten, D. (1991) Angiotensin and Cell Growth: A Link to Cardiovascular Hypertrophy. 
Journal of Hypertension, 9, 3-15. http://dx.doi.org/10.1097/00004872-199109010-00002

[9] Izumi, Y., Matsuoka, M., Kubo, A., Nakayama, T., Fukuda, N., Watanabe, Y., Soma, M. and Kanmatsuse, K. (1996) Prevention of Cardiac Hypertrophy by a Sub-Antihypertensive Dose of the $\alpha 1$-Adrenergic Antagonist Bunazosin in Dahl Salt-Sensitive Rats. Hypertension Research, 19, 147-150. http://dx.doi.org/10.1291/hypres.19.147

[10] Sato, A., Suzuki, Y., and Saruta, T. (1999) Effects of Spironolactone and Angiotensin-Converting Enzyme Inhibitor on leFt Ventricular Hypertrophy in Patients with Essential Hypertension. Hypertension Research, 22, 17-22. http://dx.doi.org/10.1291/hypres.22.17

[11] Pitt, B., Zannad, F., Remme, W.J., Cody, R., Castaigne, A., Perez, A., Palensky, J. and Wittes, J. (1999) The Effect of Spironolactone on Morbidity and Mortality in Patients with Severe Heart Failure. Randomized Aldactone Evaluation Study Investigators. The New England Journal of Medicine, 341, 709-717. http://dx.doi.org/10.1056/NEJM199909023411001

[12] Devereux, R.D. and Reichek, N. (1977) Echocardiographic Determination of Left Ventricular Mass in Man. Anatomic Validation of the Method. Circulation, 55, 613-618. http://dx.doi.org/10.1161/01.CIR.55.4.613

[13] Khosla, M.C., Page, I.H. and Bumpus, F.M. (1979) Interrelations between Various Blood Pressure Regulatory Systems and the Mosaic Theory of Hypertension. Biochemical Pharmacology, 28, 2867-2882. http://dx.doi.org/10.1016/0006-2952(79)90582-3

[14] Morgan, H.E. and Baker, K.M. (1991) Cardiac Hypertrophy: Mechanical, Neural, and Endocrine Dependence. Circulation, 83, 13-25. http://dx.doi.org/10.1161/01.CIR.83.1.13

[15] Dzau, V.J. (1993) Tissue Rennin-Angiotensin System in Myocardial Hypertrophy and Failure. Archives of Internal Medicine, 153, 937-942. http://dx.doi.org/10.1001/archinte.1993.00410080011002

[16] Grimm, D., Holmer, S.R., Riegger, G.A. and Kromer, E.P. (1999) Effects of Beta-Receptor Blockade and Angiotensin II Type I Receptor Antagonism in Isoproterenol-Induced Heart Failure in the Rat. Cardiovascular Pathology, 8, 315323. http://dx.doi.org/10.1016/S1054-8807(99)00021-6

[17] Sato, A. (1999) Effects of Spironolactone and Angiotensin-Converting Enzyme Inhibitor on Left Ventricular Hypertrophy in Patients with Essential Hypertension. Hypertension Research, 22, 17-22. http://dx.doi.org/10.1291/hypres.22.17

[18] Ferrara, A., De simone, G., Pasanisi, F., Mancini, M. and Mancini, M. (1984) Left Ventricular Mass Reduction During Salt Depletion in Arterial Hypertension. Hypertension, 6, 755-759. http://dx.doi.org/10.1161/01.HYP.6.5.755

[19] Jin, Y., Kuznetsova, T., Maillard, M., Richart, T., Thijs, L., Bochud, M., Herregods, M.C., Burnier, M., Fagard, R. and Staessen, J.A. (2009) Independent Relations of Left Ventricular Structure with the 24-Hour Urinary Excretion of Sodium and Aldosterone. Hypertension, 54, 489-495. http://dx.doi.org/10.1161/HYPERTENSIONAHA.109.130492 